\title{
A Theory of Radar Scattering by the Moon
}

\author{
T. B. A. Senior and K. M. Siegel ${ }^{1}$
}

(December 4, 1959)

\begin{abstract}
A theory is described in which the moon is regarded as a "quasi-smooth" scatterer at radar frequencies. A scattered pulse is then composed of a number of individual returns each of which is provided by a single scattering area. In this manner it is possible to account for all the major faatures of the pulse, and the evidence in favor of the theory is presented. From a study of the masured power received at different frequencies, it is shown that the scattering area nearest to the earth is the source of a specular return, and it is then possible to obtain information about the material of which the area is composed. The electromagnetic constants are derived and their significance discussed.
\end{abstract}

\section{Introduction}

Little more than a decade has now elapsed since radar contact with the moon was first established. The first reported contact was by the U.S. Signal Corps in 1946, and this was followed closely by accounts of similar success in Russia. Since then a considerable amount of effort has been expended in attempting to determine the scattering properties of the moon at radar frequencies.

The earlier experiments were limited by the equipment which was available at the time and, in consequence, many of the conclusions reached were largely qualitative. In recent years, however, the use of high-power transmitters in combination with antennas designed specifically for lunar work has provided results the accuracy of which is unquestionable, and these have shown that the scattering properties of the moon cannot be explained in terms of a "rough" scattering theory. The increased transmitted power and the improved receiver sensitivity have also permitted the use of shorter pulse lengths, and with pulses of only a few microseconds in length it has become practicable to distinguish between the returns from different parts of the lunar surface. This in turn has lead to an accurate determination of the radar distance to the moon, and the suggestion has been made that the distance should be used to obtain a more precise value for the earth's equatorial radius.

In many of these experiments a motivating force has been the possibility of employing the moon as a link in a communication system between points on the earth. The successful transmission of $\mathrm{cw}$ code was first reported by Sulzer et al. [1] ${ }^{2}$ in 1952, and subsequently by Trexler [2] who also transmitted voice. It is now well established that this type of link is a practicable proposition and signals can be communicated without appreciable loss of intelligence.

1 Radiation Laboratory, Department of Electrical Engineering, University of Michigan, Ann Arbor, Mich.

${ }_{2}^{2}$ Figures in brackets indicate the literature references on page 228 .

\section{Scattering Theories}

Optical experiments over many decades have given a fairly comprehensive picture of the major geographical features of the moon, and the general impression received is that the moon's surface is highly irregular. It is therefore natural to think of it as a rough scatterer, and since the intensity of illumination across the disk appears effectively constant, the moon has been characterized by astronomers as a "rough surface" at optical wavelengths. By this it is implied that the scattering properties of the moon can be explained in terms of a surface having a statistically uniform roughness whose scale is the order of a wavelength.

When radar reflections were first observed, it seemed reasonable to expect that these results could also be explained by a rough scattering theory. Thus, in discussing the U.S. Signal Corps experiments, DeWitt and Stodola [3] assumed without question that the moon could be treated as rough, and their analysis of the shape and intensity of the echo pulses was then based on a uniformly illuminated (and reflecting) disk. Similarly, in 1951 Kerr and Shain [4], using pulses of a millisecond or longer, concluded from their returns that "the moon is a rough reflector at this frequency $(20 \mathrm{Mc})$ and hence presumably at all higher frequencies." The same conclusion was also reached by Browne et al. [5] in 1956 from a study of the echo at $120 \mathrm{Mc}$ with 30-millisecond (msec) pulses, and it was not until shorter pulse lengths came into use that the validity of rough scattering theories was seriously questioned. It was then found that most of the return at radar wavelengths comes from only a small portion of the lunar surface, and in a summary paper published in 1957 Lovell [6] stated that "recent experiments have shown the 'uniformly bright' conclusion to be erroneous."

From an examination of pulse shapes such as those obtained by Yaplee et al. [7], the present authors felt that a scattering process similar to that for a smooth body would explain the dominant features of the returns. Thus, in 1958, a scattering 
theory was proposed [8] in which the starting point was the idea of a body which is essentially smooth as regards its radar scattering properties, and to take into account the existence of several scattering areas, the term "quasi-smooth" was applied to the lunar surface. In this paper we shall discuss the theory at some length and, in so doing, attempt to clarify those steps which, to judge from criticisms received, would appear to have caused difficulty. The fact that more experimental data has become available since the theory was originally propounded also enables us to place some of the conclusions on a somewhat firmer basis, while amending others.

\section{Fading Phenomena}

In radar returns from the moon, two distinct types of fading are apparent. The more rapid fading has a period of only a few seconds and was first observed by DeWitt and Stodola [3], and later by Kerr and Shain [4] who attributed it to the moon's libration. This has since been confirmed by Evans et al. [9] from a consideration of the diurnal variation in the fading rate. The second type of fading has a much longer period (as much as 20 to $30 \mathrm{~min}$ ) and is most clearly seen in results of experiments at the lower frequencies. It has been shown by Murray and Hargreaves [10] that these fades are consistent with the Faraday rotation of the plane of polarization produced by the earth's ionosphere. This explanation is now well accepted, but in view of the importance of the longer period fades in connection with the scattering process at the moon's surface, it is necessary to consider them in rather more detail.

\subsection{Faraday Rotation}

When a linearly polarized electromagnetic wave passes through an ionized medium in the presence of a magnetic field its plane of polarization is rotated. For a linearly polarized signal which is directed towards the moon, the polarization is first rotated on passage through the ionosphere and then, after reflection at the moon with an appropriate phase change, the polarization is again rotated on returning through the ionosphere. As the ionosphere changes, so will the rotation, and this in turn will produce a variation in the level of the signal received with an antenna of fixed linear polarization. Given a sufficient period of observation, the signal will pass through a maximum and this will occur at the same instant of time as would a minimum in an antenna of orthogonal polarization. In the extreme circumstance in which no depolarization took place at the moon's surface, this minimum would be a true zero and then, in theory at least, all the returned energy would be received by the first antenna.

The time between successive maximums or minimums is the Faraday rotation period and has been measured by many experimenters. Apart from some ambiguities it leads directly to a value for the integrated electron density along the propagation path and has been used for this purpose by, for example, Browne et al. [5].

\subsection{Fading Minimums}

The above facts are relevant to the analysis of data such as that obtained by Blevis and Chapman [11]. Using a cw system operating at a frequency of $488 \mathrm{Mc}$, the signals scattered back by the moon were received with two orthogonally polarized antennas, and samples of their data are displayed ${ }^{3}$ in figure 1 . It will be observed that when one polarization is at a maximum, the orthogonal polarization is at a minimum, and we have seen that this is a natural consequence of the Faraday rotation. More remarkable, however, is the ratio of the maximum to minimum signal levels, which is found to be approximately $16 \mathrm{db}$ [12]. A ratio as large as this is not compatible with scattering from a rough surface. Any rough surface serves to depolarize the incident field, and for a surface on which bumps are placed whose magnitude is of order one-half wavelength, the depolarization effect will be almost complete; the returns received with two ortbogonally polarized antennas will then differ by only a few decibels independently of the polarization of the transmitted field. In view of the large ratio found in the case of the moon, it can now be concluded that as regards the major sources of the scattered energy, the scattering is akin to that from a smooth surface; and in particular, the surface cannot possess a large number of (contributing) irregularities whose magnitude is comparable with the wavelength.

Although these conclusions have been reached by a study of the returns in two orthogonally polarized antennas, the same argument can be applied to the signal received on one linear polarization alone. If the maximum signal level is compared with the minimum (received at a later time), and if the interval of time is the Faraday rotation period, the ratio is again a measure of the roughness. In this respect at least, the ionosphere has a desirable effect in enabling us to dispense with one of the receiving antennas.

\subsection{An Experiment}

To emphasize the argument outlined above, an experiment was carried out in the laboratory using a sphere possessing bumps. The diameter of the sphere was $10 \lambda$ and the size of the bumps was of the order of $\lambda$. A picture of the sphere is shown in figure 2 and some of the results are given in figure 3. These can be summarized as follows:

$$
\text { Relative scattering cross section in decibels }
$$

\begin{tabular}{|c|c|}
\hline & $\sigma_{V V}$ \\
\hline Smooth sphere & \\
\hline
\end{tabular}

The cross sections for the perturbed sphere were averaged over $60^{\circ}$ in aspect. 3 The authors thank Dr. John Chapman of the Communications Laboratory,
Defence Research Telecommunications Establishment, for allowing figures 1 and 4 to be copied from presently unpublished data of Blevis and himself.

Note AdDEd IN PRoof: Since this paper was written the authors have learned that Chapman and Blevis have submitted for publication in this journal a paper eontaining the data. 
tween the polarizations is no more than $6 \mathrm{db}$. The measured difference decreases with decreasing size of bumps until the bumps become small compared with the wavelength. Accordingly, if the moon were a sphere covered with wavelength bumps, the ratio of the maximum and minimum signals in the Faraday rotation period would not exceed (about) $6 \mathrm{db}$. This is in marked contrast to the ratio which is actually observed.

\subsection{Double Fades}

The question of double fades is not directly connected with the development of our theory, but it may be of interest to discuss the matter here.

At the XIIth General Assembly of URSI at Boulder, Colorado (September 1957), Chapman pointed out that in his moon returns he sometimes obtained minimums occurring simultaneously in two orthogonally polarized receiving antennas. These phenomena were termed "double fades" and can be observed in the sample records shown in figure 4 . They were discussed in some detail at the Assembly, and both there and in later meetings were felt to violate the conservation of energy. This, however, is not so, and indeed double fades can be reproduced in the laboratory.

To explain the origin of these fades, we first employ a statement of the conservation of energy. For a fixed aspect of target, for a given transmitter polarization $\alpha$, and for all receiver polarizations $\beta$, the equation

$$
\sigma(\alpha, \beta)+\sigma\left(\alpha, \beta+\frac{\pi}{2}\right)=\text { constant }
$$

holds, where $\sigma(x, y)$ is the scattering cross section when the transmitting antenna is polarized in the direction $x$ and the receiving antenna is polarized in the direction $y$. Equation (1) is a direct consequence of conservation of energy and is an identity for all $\beta$ (see app.). It should be noted that as a function of aspect (which can be varied by rotating the body with the transmitting and receiving antennas fixed), this says nothing about whether two minimums can or cannot occur simultaneously in two orthogonally polarized receivers. Indeed, it is to be expected that for a nonsymmetrical body there will be a particular aspect such that the total backscattered energy is a minimum, and the constant in eq (1) is then a function of aspect. If at this minimum aspect we invoke the theorem represented by eq (1), we merely find that the total power received by two orthogonal antennas is independent of their polarization. Both $\sigma(\alpha, \beta)$ and $\sigma(\alpha, \beta+(\pi / 2))$ will then be small for any chosen polarization $\beta$, and both may well be smaller than they are for neighboring target aspects. In this way a double fade could occur. ${ }^{4}$ It in no way violates eq (1), nor the conservation of energy, and is not an unreasonable thing to occur with a target of asymmetrical shape.

\section{Pulse Considerations}

\subsection{Short Pulses}

Further evidence in favor of a quasi-smooth moon theory is provided by the shape of short pulse returns. When the transmitted pulse is only a few microseconds in length, the return appears to be dominated by a small number of spikes. The onset of the return is relatively sharp and the time of its occurrence is consistent with reflection from that portion of the moon which is nearest to the earth [13]. In many cases at least the spike which is associated with the leading edge of the pulse represents the peak power return.

In a series of experiments using $2-\mu$ sec pulses, Yaplee et al. [7] found that each of these spikes had a width of from 2 to $4 \mu$ sec and were separated in time by 2 to $4 \mu$ sec. The close agreement between the width of the spikes and the transmitted pulse length suggests that the source of each spike is a single scattering area. If this is so, the spikes should possess a high pulse-to-pulse correlation and a narrow power spectrum. These have been determined by Yaplee et al. [14], and by measuring the amplitude of the return as a function of distance from the leading edge it has been shown that all the first few spikes have a high correlation. The correlation decreases with increasing distance from the leading edge and, ultimately, the amplitudes assume a random distribution. As a further check the power spectra were also obtained and, as to be expected, were relatively narrow for the first few spikes.

${ }^{4}$ Since this explanation was proposed we have learned that Dr. Chapman has been thinking along similar lines,

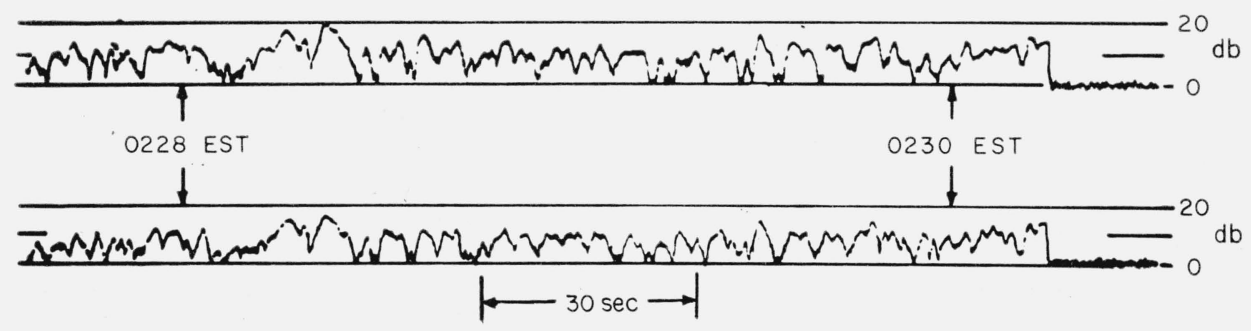

Figure 4. Lunar signals in two orthogonally polarized receivers, August 21, $195 \%$.

(The gains in the two channels have been adjusted to provide signals of comparable magnitude). 
The above results cannot be explained by a rough scattering theory, nor by one in which many scattering areas contribute towards every individual spike. On the other hand, a theory in which each leading spike is produced by a single scattering area is entirely in accordance with all the available data. The picture which now emerges has a small number of scattering areas distributed over the moon and these alone are responsible for the major features of the return. The fact that the pulse-to-pulse correlation decreases with increasing distance from the leading edge of the return is then a natural consequence of the increasing area of illumination with depth on the moon, and hence, of the increased probability of two scattering areas contributing simultaneously.

If the scattering areas were distributed over the entire moon's surface, the return from even a short pulse could persist for as much as $11.6 \mathrm{msec}$, with the last contribution coming from the areas on the limbs. In the early moon experiments the long pulse lengths and the low transmitted powers made difficult an estimate of the amount of elongation, but Kerr and Shain [4] reported an observed elongation with even millisecond pulses. In recent years, however, the use of microsecond pulses has made the actual elongation apparent, and it is now accepted that the bulk of the return is produced by only a small portion of the moon. Thus, Trexler [2] has observed that 50 percent of the power comes from the first 5 miles of depth or a circle only 105 miles in radius. This is almost exactly one tenth the radius of the moon. The most distant echoes seen were only 100 miles back. Similarly, Lovell [6] states that the main scattering takes place within an area whose radius is about one third that of the moon.

It follows that the seattering areas are not uniformly distributed over the moon's surface but are concentrated near to the center. The return from portions of the moon far from its center is relatively small and, for example, at times subsequent to the onset of the return corresponding to reflection from the limbs, the signal (in the rare cases when it has been seen) is down by 30 to $35 \mathrm{db}$ from that produced by the main scattering areas. In fact, it is only with radars such as those at Millstone Hill and Jodrell Bank that the dynamic range has been sufficient for the limb return to be detected.

\subsection{Long Pulses}

If the length of the transmitted pulse is increased, the character of the return is changed. Instead of a few major spikes superimposed upon a rough but decreasing background, there now exists a progressive buildup in the return. Given the existence of scattering areas it is to be expected that as the pulse length increases the maximum level of the return will also increase due to the simultaneous illumination of more and more areas. On the other hand, because of the concentration of these areas near the center of the moon, the increase cannot continue indefinitely, and ultimately a pulse length is reached at which the power return is the same as that ob- tained with $\mathrm{cw}$. This behavior is indeed found. With a long transmitted pulse the leading edge of the return is still sharp and again corresponds to scattering from that portion of the moon which is nearest to the earth, but while this area is still being illuminated the return from the next scattering area comes in and interferes with the first. As time progresses more returns appear, and these will interfere to produce a variety of peaks which are superimposed upon an increasing background level. This will continue for a time equal to the transmitted pulse length, or until the cw return has been achieved, whichever is the sooner. With increase of time beyond the transmitted pulse length, the level of the return decreases and disappears into noise after (about) $200 \mu \mathrm{sec}$. In a return of this type all the peaks other than the first must be attributed to interference.

As stated above, an increase in the pulse length is usually accompanied by an increase in the peak power return. Conversely, a decrease in the pulse length is generally reflected by a decrease in the return, and this leads to the concept of modulation loss. The modulation loss was first introduced by Trexler [2] and is essentially a measure of the reduction in the peak power return when using finite pulse lengths, as compared with the level of the cw return. Based upon many experiments at different frequencies using a variety of pulse lengths, Trexler has determined values for the loss. These have been communicated by Youmans [15] and show a modulation loss that increases from $1 \mathrm{db}$ for a pulse length of $200 \mu \mathrm{sec}$, through $8.5 \mathrm{db}$ at $30 \mu \mathrm{sec}$, and $17 \mathrm{db}$ at $10 \mu \mathrm{sec}$, to $22 \pm 2 \mathrm{db}$ at $2 \mu \mathrm{sec}$. The number of points is not enough to describe the precise variation at small pulse lengths, but it would appear that the loss is effectively constant for pulse lengths less than approximately $5 \mu$ sec. With pulses of this duration, the contribution from the first scattering area is isolated from the rest. In contrast, pulses of $300 \mu \mathrm{sec}$ are sufficient to realize the peak cw return.

\subsection{Scattering Areas}

In order to estimate the number of major scattering areas two different methods are available. The first of these consists of merely counting the larger peaks in a short pulse return. With this approach the number obtained lies somewhere between 20 and 30, but inaccuracies do arise because of interference between signals from scattering areas in the same annular regions of the moon. In other words, a one-to-one correspondence between peaks and scattering areas may not exist for all portions of a returned pulse.

The second method is based upon the modulation loss and involves a detailed examination of the power levels used. To begin with we remark that the measured values for the modulation loss were found by restricting the times of observation to ones at which an appreciable signal is received. Such a statement has many ramifications and not only implies good propagation conditions, but a straight average over time. Thus, the cw level which is employed as a reference is an average over time for those 
periods at which a large return appears. This level exceeds the one corresponding to random excitation of the various scattering areas.

As regards the pulse returns, the power level used is the average over pulses of the maximum for each. With very short pulses ( 2 to $5 \mu$ sec, say), this average is merely the return from the first scattering area. As the pulse length increases more areas are illuminated, but with short pulses at least the average peak level is almost that appropriate to in-phase addition of the individual returns. Naturally, the amount by which it falls below the in-phase level increases with increasing pulse length, but because the scattering areas are not large in number, this difference never becomes more than (about) $7 \mathrm{db}$. In consequence, the average peak level remains considerably above the level corresponding to random excitation. The figure $7 \mathrm{db}$ was obtained from results at 201 and 915 Mc using millisecond pulses [16] and represents the difference between the largest signal observed and the mean level in the sense described above. It is natural to imagine that this large return occurred when the individual signals were precisely in-phase, and the assumption that this was so provides an estimate for the number of major scattering areas. For this purpose we assume that each scattering area gives rise to a signal of the same magnitude and, in addition, that the peak return from a $2-\mu$ sec pulse comes from a single area. All the areas together then produce a peak (or in-phase) return which is $22+7=29 \mathrm{db}$ larger than that from one, indicating that there are 28 areas in toto. The result is in good agreement with that obtained by direct counting of spikes, and this is in spite of the inaccuracies inherent in both methods. A more detailed discussion of the subject is out of place here, but the above arguments are the basis for our present belief that the number of major scattering areas is between 20 and 30 .

No statement has yet been made about the physical nature of the scattering areas. Although much work remains to be done in this connection, some information can be obtained by an extension of the above analysis. If the modulation loss curve is modified in such a way as to give results appropriate to in-phase addition, it is possible to estimate the variation in the total number of scattering areas in an annular region for which the slope of the mean lunar surface changes by a fixed amount (for example, $1^{\circ}$ ) from the tangent plane at the nose. It is found that the number of areas per degree of slope decreases uniformly with increase of slope beyond about $2^{\circ}$. This occurs in spite of the increasing area of these regions and suggests that whereas the specular areas nearest the center of the moon are portions of spherical surfaces, the areas further back are possibly craterlike. As the depth increases the decreasing probability of finding surfaces suitably alined is the reason why the level of a short pulse return falls below its peak by 30 or more decibels after $600 \mu$ sec (approx) from the onset of the return. In effect, therefore, all the scattering areas are located within $300 \mu$ sec of the center of the moon, corresponding to a depth of 30 miles.

\subsection{Pulse Elongation}

A direct consequence of the existence of many scattering areas is the elongation of any pulse returned from the moon. We have seen that this can be as much as $600 \mu$ sec and, indeed, if a short pulse return is examined, the general impression received is of a few major spikes superimposed on a rough but decreasing background. This background will be called a "tail" and its general features are the same for pulses of any length. The peaks have already been discussed in some detail, and we shall now consider possible mechanisms which could produce other pulse distortion and, in particular, generate a tail.

It is first remarked that for a smooth sphere of radius $a$ the return from an incident pulse has a tail which is a function of both the pulse length and the wavelength. If, for example, the pulse length is of order $a$ and, in addition, $2 \pi a / \lambda$ has a value near to unity, the analysis given in [19] shows that a tail will persist with appreciable amplitude for a considerable length of time. For a body which has many scattering areas it is to be expected that the relevant dimension is the size of the scattering areas, and if this is now comparable with the wavelength, each individual return may well possess a tail. The precise amount of tail will depend upon the type of scattering object, but since the dimensions of the major scattering areas on the surface of the moon are almost certainly measured in tens (or hundreds) of wave-lengths, this effect is unlikely to be significant.

In practice, there are many other effects which are almost certainly more important. Any roughness of the lunar surface will produce a residual signal and, in addition, pulse degradation will be caused by differential action on the frequency components by the propagation medium (for example, the Faraday rotation is frequency dependent). Finally, there is the limited receiver bandwidth to be considered. In most of the experiments the bandwidth has been less than the optimum for the pulse length, and, mathematically at least, this could be the source of a large amount of the tail. Taking, for example, the experiments carried out by Yaplee et al. [7] using $2 \mu$ sec pulses, a straightforward calculation shows that the bandwidth of $350 \mathrm{kc}$ could generate a tail which only decays to 6 percent of the peak after 16.7 usec. Under these circumstances, a detailed discussion of the tail contributions is somewhat academic.

\section{The Measured Power Return}

In the last few years many measurements have been made of the transmission loss in radar scattering by the moon, and from these results the scattering cross section can be obtained. The quantity of data is now increasing rapidly, and the results which are available at this time are listed in table 1 . The cross sections are there expressed as fractions of $\pi a^{2}$ with $a=1.74 \times 10^{6} \mathrm{~m}$. It will be recognized that $\pi a^{2}$ is the cross section of a perfectly conducting sphere 
TABLE 1

\begin{tabular}{|c|c|c|c|}
\hline & Wavelength & $\sigma / \pi a^{2}$ & $\begin{array}{l}\text { Probable error } \\
\text { (where known) }\end{array}$ \\
\hline $\begin{array}{l}\text { (i) } \\
\text { (ii) } \\
\text { (iii) } \\
\text { (iv) } \\
\text { (v) } \\
\text { (vi) }\end{array}$ & $\begin{array}{c}m \\
0.10 \\
.14 \\
.33 \\
.61 \\
.73 \\
.75\end{array}$ & $\begin{array}{l}4 \times 10^{-4} \\
3 \times 10^{-4} \\
9 \times 10^{-2} \\
3 \times 10^{-2} \\
7 \times 10^{-2} \\
1 \times 10^{-1}\end{array}$ & $\begin{aligned} & d b \\
& \pm 4 \text { or } 5 \\
& \pm 3 \\
& \pm 3 \\
& \pm 3 \\
& \pm 3\end{aligned}$ \\
\hline $\begin{array}{r}\text { (vii) } \\
\text { (viii) } \\
\text { (ix) } \\
\text { (x) } \\
\text { (xi) } \\
\text { (xii) }\end{array}$ & $\begin{array}{l}1.00 \\
1.49 \\
1.50 \\
1.99 \\
2.5 \\
3.0\end{array}$ & $\begin{array}{r}(5 \sim 9) \times 10^{-2} \\
7 \times 10^{-2} \\
(6 \sim 10) \times 10^{-2} \\
5 \times 10^{-2} \\
1 \times 10^{-1} \\
1 \times 10^{-1}\end{array}$ & $\begin{array}{l} \pm 4 \\
\pm 3 \\
\pm 4 \\
\pm 3 \\
\pm 3\end{array}$ \\
\hline
\end{tabular}

whose radius is that of the moon. In interpreting the data an essential factor is the pulse length. Whereas the measurements at 3,000 Mc were carried out with pulse lengths of 5 and $2 \mu$ sec, all the others were based on an analysis of cw (or verv long pulse) returns, and this accounts for the significantly lower values in (i) and (ii).

Of the above values of $\sigma$, the first was obtained by Hey and Hughes [17] of the Royal Radar Establishment at a frequency of $3,000 \mathrm{Mc}$ using 5 - $\mu$ sec pulses. The second is due to Yaplee et al. [7] of the U.S. Naval Research Laboratory, and is for 2,860 Mc with $2-\mu$ sec pulses. The third and eighth were found by Aarons et al. [16] of the Air Force Cambridge Research Center using millisecond pulses at frequencies of 915 and $201 \mathrm{Mc}$, respectively. The fourth measurement ${ }^{5}$ was made by Blevis and Chapman [11] of the Defence Research Telecommunications Establishment at a frequency of 488 Mc using a cw system, and the fifth is also a cw result obtained by Fricker et al. [18] of Lincoln Laboratory with a frequency of $413 \mathrm{Mc}$.

The sixth and twelfth values were found by Leadabrand [19] of the Stanford Research Institute using millisecond pulses at frequencies of 400 and $100 \mathrm{Mc}$, respectively. The seventh is due to Trexler [2] of the U.S. Naval Research Laboratory and has been deduced from his statement that the cw transmission loss is $258 \mathrm{db}$ when calculated on the basis of isotropic transmitting and receiving antennas. The two values given for $\sigma$ in (vii) and (ix) are for a moon at perigee and apogee, respectively. The ninth result is the average obtained from many cw measurements made at or near a frequency of $200 \mathrm{Mc}$, and has been stated by Trexler [2] as a transmission loss of $254 \mathrm{db}$. The tenth measurement was made by Webb [20] of the University of Illinois at a frequency of $151 \mathrm{Mc}$ using a cw system. The eleventh result was obtained by Evans [21] of Jodrell Bank with a pulse length of $30 \mathrm{msec}$ at a frequency of $120 \mathrm{Mc}$, and is a refinement of that originally measured by Browne et al. [5].

In addition to the above there is a "summary measurement" which indicates the general dependence of the cross section on wavelength. From a review of the data available to him, Trexler [2]

${ }^{5}$ Note AdDED IN PROOF: We have been informed by Dr. Chapman that a more detailed study of their data has increased their value for the cross section of the moon to $5 \times 10^{-2} \pi \mathrm{a}^{2}$. This has the effect of making still better the agreement between theory and experiment (see fig. 5). states that for frequencies between 20 and 3,000 Mc the average cw transmission loss increases at a rate of $6 \mathrm{db}$ per octave, passing through the value $258 \mathrm{db}$ at $300 \mathrm{Mc}$. Reference to the radar equation shows that this implies a cross section which is wavelength independent.

A meaningful comparison of all the available data can only be carried out if the cross sections can be reduced to those which are appropriate to a common pulse length. It will be observed that most of the values for $\sigma$ were obtained from either $\mathrm{cw}$ or long pulse experiments, and as such they represent the power contributed by many scattering areas. To discuss the properties of a single scattering area, it is necessary to know the power associated with one area alone, and this is only the peak return in the case of very short pulses. It would clearly be desirable to confine the analysis to data which was actually obtained with pulses of this length, but since the number of these experiments is still very small, we are forced to make use of all available data. To do this, "correction factors" are applied to the scattering cross sections to reduce them to the values for microsecond pulses. Using Trexler's figure for the modulation loss associated with a pulse of duration $5 \mu$ sec or less, the modified data is then as follows:

$\begin{array}{rll}\text { (i) } & \lambda=0.10 \mathrm{~m}, & \sigma=4 \times 10^{-4} \pi a^{2} \\ \text { (ii) } & \lambda=.14 \mathrm{~m}, & \sigma=3 \times 10^{-4} \pi a^{2} \\ \text { (iii) } & \lambda=.33 \mathrm{~m}, & \sigma=5.4 \times 10^{-4} \pi a^{2} \\ \text { (iv) } & \lambda=.61 \mathrm{~m}, & \sigma=1.9 \times 10^{-4} \pi a^{2} \\ \text { (v) } & \lambda=.73 \mathrm{~m}, & \sigma=4.3 \times 10^{-4} \pi a^{2} \\ \text { (vi) } & \lambda=.75 \mathrm{~m}, & \sigma=6.3 \times 10^{-4} \pi a^{2} \\ \text { (vii) } & \lambda=1.00 \mathrm{~m}, & \sigma=4.7 \times 10^{-4} \pi a^{2} \\ \text { (viii) } & \lambda=1.49 \mathrm{~m}, & \sigma=4.2 \times 10^{-4} \pi a^{2} \\ \text { (ix) } & \lambda=1.50 \mathrm{~m}, & \sigma=5.3 \times 10^{-4} \pi a^{2} \\ \text { (x) } & \lambda=1.99 \mathrm{~m}, & \sigma=3.0 \times 10^{-4} \pi a^{2} \\ \text { (xi) } & \lambda=2.5 \mathrm{~m}, & \sigma=6.3 \times 10^{-4} \pi a^{2} \\ \text { (xii) } & \lambda=3.0 \mathrm{~m}, & \sigma=6.3 \times 10^{-4} \pi a^{2}\end{array}$

These results are interpreted as the scattering cross section of the first scattering area, and are plotted in figure 5. Unfortunately it is difficult to estimate their accuracy. Even with the original data the probable error is not always known, but where it is it has been noted in table 1 . It is seen that a typical error is $\pm 3 \mathrm{db}$. In addition to these inaccuracies there are those which are inherent in trying to deduce the results of a short pulse experiment from a long pulse or cw measurement. In the absence of more definite information, we have therefore associated an uncertainty of $\pm 3 \mathrm{db}$ with all the modified data, and have denoted these limits by arrows in figure 5. It would seem likely that this is an underestimate, but nevertheless the consistency of the results gives added faith in both the original data and in Trexler's measurement of the modulation loss.

\section{Theoretical Considerations}

To obtain a theoretical expression for the cross section of the first scattering area, it is necessary to know the physical shape of the scatterer and also the material of which it is composed. In the original exposition of the quasi-smooth moon theory [8], it was 


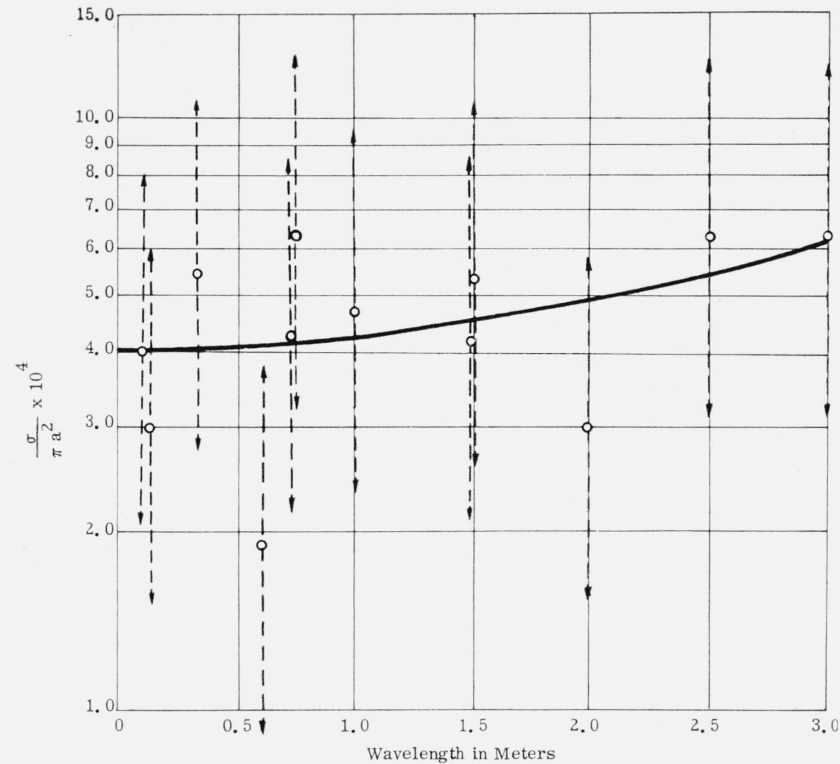

Figure 5. Comparison between theory and experiment.

assumed that the return from this area is a specular contribution, but the suggestion has been made that facets, or even conelike projections, should also be considered as sources of return. To allow for this possibility, a formula for the cross section will now be derived of sufficient generality to embrace several types of scatterer.

\subsection{Basic Formulas}

Let us consider first a perfectly conducting body of arbitrary convex shape on which a plane wave is incident. It will be assumed that the receiver is in the far field of the body regarded as a scatterer.

Since the body is perfectly conducting, the reflection coefficient is unity, and the leading term in the asymptotic expansion of the cross section for small wavelengths is given by geometrical optics. If the body has no corners or facets perpendicular to Poynting's vector, the geometrical optics cross section is wavelength independent and is simply

$$
\sigma=\pi R_{1} R_{2}
$$

where $R_{1}$ and $R_{2}$ are the principal radii of curvature of the specular region. For backscattering, the center of the specular region is that point on the body which is nearest to the receiver. In the particular case in which $R_{1}=R_{2}=a$, eq (2) reduces to

$$
\sigma=\pi a^{2}
$$

which will be recognized as the cross section of a perfectly conducting sphere of radius $a$.

If the body is not smooth and convex, but is still perfectly conducting, other portions may contribute towards the scattering cross section, and while small scale irregularities will not be considered, any large scale perturbation must be taken into account. Falling into this category is a corner reflector or large flat area, the cross section of which is

$$
\sigma=\frac{A}{\lambda^{2}}
$$

where $A$ is proportional to the square of the area. For positions of the receiver in the direction of peak return, $A$ is essentially wavelength independent. The equivalent structure on the surface of the moon could be a smooth mountain face taken either by itself (to give a flat area) or in combination with the level lunar surface (to produce a corner reflector). Because of the narrow beamwidth, it seems unlikely that such a scatterer could provide a significant contribution to the actual moon's return over any range of aspects, but in the interests of generality the possibility will not be excluded.

A second type of structure is a conelike projection of small included angle (the lunar equivalent might be a mountain peak), and the cross section of this is

$$
\sigma=C \lambda^{2}
$$

where $C$ is independent of the wavelength. If both types of scatterer are present on the body, the total cross section can be written in the form

$$
\sigma=\frac{A}{\lambda^{2}}+B+C \lambda^{2}
$$

with $A, B, C \gg 0$. In this expression, $B$ represents the geometrical optics cross section of the unperturbed body.

It will be appreciated that in writing down eq (6) we have neglected interference between the individual scatterers. In its application to the moon, the equation is only required in the analysis of the leading part of the pulse, and to contribute towards this part all the relevant scatterers must lie in the same general area. The interference which then exists will show up as a fluctuation in the power level of individual pulses as a function of aspect. Since no fluctuation appears which can be attributed to this effect, it seems likely that one type of scatterer and, moreover, only one scatterer of that type, is responsible for the major contribution towards the leading part of the pulse. Accordingly, if the cross section in eq (6) is compared with the experimental data, we should expect to find that one of $A, B$, and $C$ dominates the other two.

Unfortunately, eq (6) is not immediately applicable to the analysis of the data. In the first place, the moon is not perfectly conducting nor is it likely to be homogeneous. In addition, many of the experiments have been carried out using beamwidths which do not always illuminate the whole moon and, finally, there is the near-zone effect. As pointed out in reference [8] the distance between the earth and the moon is substantially less than $4 \pi a^{2} / \lambda$, where $a$ is the moon's radius, so that any receiver on the earth 
will necessarily be within the near zone of the moon regarded as a scatterer. The effect, if any, of these conditions upon the scattering cross section will now be considered.

\subsection{Near-Zone Effect}

After it was appreciated that the earth is in the near zone of the moon at all frequencies which will penetrate the ionosphere, Dr. V. H. Weston of this laboratory obtained the leading term in the small wavelength expansion for the cross section of a perfectly conducting sphere with transmitter and receiver located arbitrarily.

Taking the transmitter to be a point source at a distance $r_{1}$ from the nearest point on the surface of the sphere and the receiver at a distance $r_{2}$ from the same point and in the same direction, Weston's analysis [22] yields a cross section

$$
\sigma=4 \pi\left(\frac{a r_{1} r_{2}}{a\left(r_{1}+r_{2}\right)+2 r_{1} r_{2}}\right)^{2}
$$

where $a$ is the radius of the sphere. There are now two distinct cases that can be treated. If the transmitter and the receiver are at the same position so that $r_{1}=r_{2}(=r$, say), eq ( 7$)$ becomes

$$
\sigma=\pi\left(\frac{a r}{a+r}\right)^{2} .
$$

When $a>>r$,

$$
\sigma=\pi r^{2},
$$

which is therefore the cross section of a sphere for a transmitter and receiver near to the surface and also the cross section of an infinite flat plate for a transmitter and receiver anywhere, and when $a<<r$, eq (8) gives

$$
\sigma=\pi a^{2} .
$$

A second set of results can be deduced from eq (7) when the incident field is a plane wave. By taking the limit of (7) as $r_{1} \rightarrow \infty$ (it is sufficient that $r_{1}>>$ $\left.a, r_{2}\right)$, we have

$$
\sigma=4 \pi\left(\frac{a r_{2}}{a+2 r_{2}}\right)^{2}
$$

and for $a>>r_{2}$

$$
\sigma=4 \pi r_{2}^{2}
$$

which differs by a factor 4 from the corresponding result for spherical wave illumination. Of course, when $r_{2}>>a$, eq (9) reduces to

$$
\sigma=\pi a^{2}
$$

in agreement with eq (3).

The above analysis shows that for cw illumination the leading term in the expansion for the cross section of a sphere is $\pi a^{2}$ for both plane and spherical waves, and is independent of the position of the receiver providing its distance from the sphere is large compared with the radius. In reference [22] it is also shown that the same formula gives the scattering cross section which would be obtained with pulses of any length.

These results have an obvious interpretation in terms of the cross section of the specular area, and in order to realize the full cross section it is only necessary for the incident field to cover this particular area. In consequence, the beam width can be appreciably less than the angle subtended by the sphere without affecting the peak return. Moreover, because the moon's return is given by a finite number of scattering areas, we need only illuminate these to obtain the total power return, and since the areas are confined to a region about 500 miles in extent, a beamwidth of approximately $8 \mathrm{~min}$ of arc should be sufficient to realize all the available power.

\subsection{Reflection Coefficient}

The formulas which have been obtained so far only apply to perfectly conducting bodies, and we shall now consider the effect of taking into account the material constants of the body.

For a body which is not perfectly conducting but is composed of a homogeneous isotropic material, the leading term in the small wavelength expansion differs from the optics cross section by a factor which depends upon the electromagnetic constants of the surface and is, in fact, the power reflection coefficient $|R|^{2}$. In the case of backscattering from a flat plate or specular area, the maximum return occurs at normal incidence, and since the dimensions of the scatterer are large compared with the wavelength, the reflection coefficient is the same as for an infinite slab of the material.

If a plane electromagnetic wave is incident normally on such a slab,

$$
R=\frac{1-\sqrt{\frac{\mu_{0}}{\mu}\left(\frac{\epsilon}{\epsilon_{0}}+i \frac{s}{\omega \epsilon_{0}}\right)}}{1+\sqrt{\frac{\mu_{2}}{\mu}\left(\frac{\epsilon}{\epsilon_{0}}+i \frac{s}{\omega \epsilon_{0}}\right)}}
$$

(see, for example, reference [23]), where $\epsilon, \mu$, and $s$ are the permittivity, permeability, and conductivity, respectively, of the material. The subscript "o", denotes the same quantities for free space and $\omega$ is the frequency. Rationalized mks units are employed with a time dependence $\exp (-i \omega t)$.

If the material is primarily dielectric in behavior at the relevant frequencies (as seems probable for the moon), then

and

$$
s<<\omega \epsilon
$$

$$
R \simeq \frac{1-b-i b^{\prime}}{1+b+i b^{\prime}}
$$

where

giving:

$$
b=\sqrt{\frac{\mu_{0} \epsilon}{\mu \epsilon_{0}}} \text { and } \quad b^{\prime}=\frac{s}{2 \omega \epsilon_{0}} \sqrt{\frac{\mu_{0} \epsilon_{0}}{\mu \epsilon}} \text {, }
$$

$$
|R|^{2} \simeq \frac{(1-b)^{2}+b^{\prime 2}}{(1+b)^{2}+b^{\prime 2}}
$$


In the denominator the second term is certainly small compared with the first, and hence

$$
|R|^{2} \simeq\left(\frac{1-b}{1+b}\right)^{2}+\left(\frac{b^{\prime}}{1+b}\right)^{2} .
$$

Since $\omega=2 \pi c / \lambda$, this equation can be written as

with

$$
|R|^{2} \simeq d+\lambda^{2} d^{\prime}
$$

$$
d=\left(\frac{1-b}{1+b}\right)^{2}
$$

and

$$
d^{\prime}=\left(\frac{b}{1+b} \frac{s}{4 \pi c \epsilon}\right)^{2}
$$

It seems reasonable to assume that $d$ will not be identically zero.

For a flat plate or specular area the cross section for infinite conductivity must be multiplied by the reflection coefficient $|R|^{2}$, and while the values of $d$ and $\mathrm{d}^{\prime}$ may be different for each individual scatterer, no confusion should arise if distinguishing marks are omitted.

In the case of scattering by a conelike projection, the reflection coefficient cannot be obtained from a consideration of normal incidence on an infinite slab, but from a study of the complete optics solution for a cone of imperfect conductivity, the corresponding expression for $|R|^{2}$ is found to have the same wavelength dependence as appears in eq (11). Although the coefficients are no longer given by eqs (12) and (13), a detailed derivation is unnecessary in view of the conclusions reached in section 7.1. If the same symbols $d$ and $d^{\prime}$ are used to denote these coefficients, the combined cross section of the various types of scatterers can be written in the following general form:

$$
\sigma=\frac{A d}{\lambda^{2}}+\left(A d^{\prime}+B d\right)+\left(B d^{\prime}+C d\right) \lambda^{2}+C d^{\prime} \lambda^{4} .
$$

In order to apply this result to the analysis of the data, it is necessary to assume that $d$ and $d^{\prime}$ are independent of wavelength. For the material of which the moon's surface is composed it seems reasonable to assume that $\epsilon, \mu$, and $s$ do not vary rapidly as a function of frequency, and the expression for $\mid R^{2}$ can then be used providing the material of the first specular area can be regarded as homogeneous. In particular, there is no requirement that the whole moon should be effectively homogeneous.

\section{Comparison With the Data}

The experimental data for the scattering cross section of the first specular area will now be used to determine the most probable values for the constants which appear in eq (14). In this way it is possible not only to determine the dominant type of scatterer which is responsible for the return, but also to obtain information about the material of which the scattering area is composed.

\subsection{Least Squares Analysis}

Over the range of wavelengths for which the data is available, the variation in the cross section is relatively small, and this suggests that the dominant term in eq (14) is wavelength independent. This is confirmed by a least squares analysis in which the above expression for $\sigma$ is compared with the measured data. The values obtained for the terms are

$$
\begin{aligned}
A d & =-8.94 \times 10^{-7} \times \pi a^{2} \mathrm{~m}^{4}, \\
A d^{\prime}+B d & =4.45 \times 10^{-4} \times \pi a^{2} \mathrm{~m}^{2}, \\
\left(B d^{\prime}+C d\right) & =-1.03 \times 10^{-5} \times \pi a^{2} \text { dimensionless, } \\
\left(C d^{\prime}\right) & =3.71 \times 10^{-6} \times \pi a^{2} \mathrm{~m}^{-2},
\end{aligned}
$$

where $a=1.74 \times 10^{6} \mathrm{~m}$. In the range of $\lambda$ for which we have experimental values we find that the second term exceeds all others and for the smaller wavelength experimental points the second term exceeds all others by at least an order of magnitude. In particular, $A d$ is both small and negative, and can therefore be neglected. Since $d \neq 0$, it follows that $A$ can be taken zero, and this rules out the possibility of facets making a major contribution towards the measured values of $\sigma$. In addition, $C d^{\prime}$ is extremely small, and although this does not determine that $C$ itself is negligible, it does enable us to remove a further wavelength dependence from the formula for $\sigma$.

By repeating the least squares analysis using only the $\lambda^{0}$ and $\lambda^{2}$ terms, it is found that

$$
\begin{aligned}
B d & =4.04 \times 10^{-4} \times \pi a^{2} \mathrm{~m}^{2} \\
\left(B d^{\prime}+C d\right) & =2.37 \times 10^{-5} \times \pi a^{2} \text { dimensionless. }
\end{aligned}
$$

The specular area still provides the dominant contribution and since neither $B d^{\prime}$ nor $C d$ can be negative, the effect of any conelike projection is smaller by at least a factor of 17 for $\lambda<1 \mathrm{~m}$. On the other hand, no argument of this type can distinguish between the return from such a projection and the conductivity effect of the first specular area. As previously remarked, a variation in amplitude in the leading part of pulses could indicate the existence of more than one scatterer, but the fact that no variation exists only precludes the possibility of two or more scatterers giving comparable returns. In the present case we have already seen that the specular area easily outweighs all the other scatterers, and on the ground that a single scattering object provides the most attractive picture, the assumption will be made that $C$ can be neglected. It should be noted that the presence (or absence) of any conelike contribution in no way affects the value of $B d$.

The above assumption implies that the leading part of a pulse comes from a single specular area, 
and since the scattering cross section is then

$$
\sigma=\pi R_{1} R_{2}|R|^{2},
$$

where $R_{1}$ and $R_{2}$ are the radii of curvature, the lack of any appreciable pulse-to-pulse variation now suggests that the magnitudes of $R_{1}$ and $R_{2}$ are the same for every specular area which is responsible for the return. The only radius which appears to have an a priori probability of occurring is the moon's radius itself. It is therefore assumed that both radii of curvature are equal to the mean lunar radius, and while this must remain a possible source of error, an alternative choice of radii is discussed in section 7.3 .

Replacing $R_{1}$ and $R_{2}$ by $a$, the cross section becomes

$$
\sigma=\pi a^{2}|R|^{2}
$$

in agreement with that used in reference [8]. From eqs (15) and (16) we then have

$$
\sigma=\left(d+\lambda^{2} d^{\prime}\right) \pi a^{2}
$$

with $d=4.04 \times 10^{-4}$ and $d^{\prime}=2.37 \times 10^{-5} \mathrm{~m}^{-2}$, and the corresponding curve is plotted in figure 5 .

\subsection{Electromagnetic Constants}

The electromagnetic constants of the material of which the specular area is composed can be calculated from the above values of $d$ and $d^{\prime}$. If these are inserted into eqs (12) and (13), it is found that

$$
\begin{aligned}
& \epsilon / \mu=6.5 \times 10^{-6} \mathrm{mhos}^{2}, \\
& s / \mu=2.4 \times 10^{2} \mathrm{mhos} / \text { henry, }
\end{aligned}
$$

or

$$
\begin{aligned}
& \epsilon / \mu=7.6 \times 10^{-6} \text { mhos }^{2}, \\
& s / \mu=2.7 \times 10^{2} \text { mhos/henry. }
\end{aligned}
$$

The two sets appear because of the ambiguity in sign in taking the square root of $d$. Aesthetically at least, it is more pleasing to select that set for which the ratio $\epsilon / \mu$ exceeds its value for free space, and this is the second of those given above. We note in passing that only a preliminary version of the first based on seven experimental points was given in references [8] and [24].

From a power measurement alone, $\epsilon$ and $s$ cannot be obtained explicitly, but if it is assumed that $\mu=\mu_{0}$, then

$$
\begin{aligned}
& \epsilon=9.6 \times 10^{-12} \text { farads } / \mathrm{m}\left(=1.1 \epsilon_{0}\right) \\
& s=3.4 \times 10^{-4} \text { mhos } / \mathrm{m} .
\end{aligned}
$$

Both of these values are smaller than expected and few (if any) naturally occurring substances on earth, apart from liquids or gases, have a relative permittivity as low as 1.1. Although this is no reason for ruling out the possibility of an appropriate lunar substance, it may be of interest to observe that if $\mu$ is increased by a factor $\alpha, \epsilon$, and $s$ are increased by the same amount. Taking $\alpha$ to be, for example, 1.4, we now have

$$
\begin{aligned}
\mu & =1.4 \mu_{0}, \\
\epsilon & =1.5 \epsilon_{0}, \\
s & =4.8 \times 10^{-4} \operatorname{mhos} / \mathrm{m},
\end{aligned}
$$

and these are not inconsistent with soils such as magnetite.

\subsection{Discussion}

Over the range of wavelengths from 0.1 to $2.5 \mathrm{~m}$ the variation in the measured cross section is relatively small but is accurately reproduced by the wavelength dependence of the formula for $|R|^{2}$. In the authors' opinion, this variation is significant and implies a small (but nonzero) conductivity for the material of which the specular area is composed. On the other hand, Purcell [25] has pointed out that a cross section which is wavelength independent can be chosen to fit the data to within $\pm 3 \mathrm{db}$. A cross section of this type would be represented by a line parallel to the abscissa in figure 5 and would imply either a material of zero conductivity or one whose conductivity varied inversely as the wavelength. For this frequency range at least, the cross section would be approximately $3.5 \times 10^{9} \mathrm{~m}^{2}$.

Purcell has also suggested that in eq (17) the radii of curvature should be about a tenth of the lunar radius, thereby increasing $|R|^{2}$ by a factor of (approximately) $10^{2}$. This now agrees with the reflection coefficient of certain dry sands on earth, and even with $\mu=\mu_{0}$ the resulting permittivity is increased to $1.5 \epsilon_{0}$.

Unfortunately, the decreased radius of curvature has several unpleasant consequences. In a short pulse return from the moon, the second major peak is similar to the first in all important respects. To a somewhat lesser extent, this is also true of the third and fourth peaks and, in particular, the power levels do not differ appreciably. Purcell's picture then requires that each peak be produced by a region whose radii of curvature are less than that of the lunoid. Instead of a single bump in the center of the moon, we are now forced to postulate a series of undulations of amplitude $a / 10$, and these have to extend over a considerable portion of the lunar surface. It seems unlikely that such a system of (smooth) undulations would have escaped detection in lunar photographs.

If, on the other hand, the radii of curvature are equal to the radius of the moon, the low permittivity may have to be accepted, though the possibility that $\mu$ exceeds $\mu_{0}$ should still be borne in mind. However, the authors are not as yet convinced that a relative permittivity as low as 1.1 is necessarily inconsistent with a solid material in the environment of the moon, and feel that selected powders might conceivably fit the bill. 


\section{Conclusions}

The theory of a quasi-smooth moon was arrived at from a study of many sets of experimental data, and it is felt that the pulse shapes and power returns can be satisfactorily explained on this basis. It is found that the major scattering areas are concentrated near to the center of the moon, and their total number appears to be approximately 25 . An indication of their distribution can also be obtained, but no attempt has been made to associate these areas with individual features on the moon. There is clearly scope for further work in this connection.

The area nearest to the earth provides a specular return, and if pulses of short duration are employed, this return shows up as a peak which can be distinguished from the remainder of the pulse. Only a scattering process of this type could produce the high pulse-to-pulse correlation and the lack of wavelength dependence observed in the leading peak. It seems probable that the adjacent scattering areas are also specular in character, but no statement is yet possible about the areas as a whole.

The power level of the leading peak now determines the scattering cross section of the area nearest to the earth, and this in turn specifies the electromagnetic constants for the material of which the area is composed. Using the measured power returns at a variety of different wavelengths the ratios of permittivity to permeability and conductivity to permeability for the surface have been found. The values are smaller than expected, but are not necessarily inconsistent with certain powdered materials in the environment of the moon. In this connection, laboratory experiments under vacuum conditions will be carried out in the near future to determine the constants for several substances whose occurrence on the moon has been postulated.

The analysis reported in this paper and, indeed, the development of the theory itself, were only possible because of the experimental data made available to us. We therefore express our gratitude to the many experimenters who freely communicated their results to us even prior to publication elsewhere. We are also indebted to members of the Radiation Laboratory, among whom should be mentioned R. E. Hiatt, who performed the experiment described in section 3.3, and W. E. Fensler, who provided assistance with this manuscript. The work described herein was carried out for the National Aeronautics and Space Administration under Grant $\mathrm{NaG}-4-59$.

\section{References}

[1] P. G. Sulzer, G. F. Montgomery, and I. H. Gerks, An uhf moon relay, Proc. I.R.E. 40, 361 (1952).

[2] J. H. Trexler, Lunar radio echoes, Proc. I.R.E. 46, 286 (1958).

[3] J. H. DeWitt, Jr., and E. K. Stodola, Detection of radio signals reflected from the moon, Proc. I.R.E. 37, 229 (1949).
[4] F. J. Kerr and C. A. Shain, Moon echoes and transmission through the ionosphere, Proc. I.R.E. 39, 230 (1951).

[5] I. C. Browne, J. V. Evans, J. K. Hargreaves, and W. A. S. Murray, Radio echoes from the moon, Proc. Phys. Soc. B 69, 901 (1956)

[6] A. C. B. Lovell, Report on British work carried out in radio astronomy since 1954 , presented at XIIth General Assembly of URSI, Boulder, Colo. (1957).

[7] B. S. Yaplee, R. H. Bruton, K. J. Craig, and N. G. Roman, Radar echoes from the moon at a wavelength of $10 \mathrm{~cm}$., Proc. I.R.E. 46, 293 (1958).

[8] T. B. A. Senior and K. M. Siegel, Radar reflection characteristics of the moon, Paris Symp. on Radio Astron. (ed. R. N. Bracewell) Stanford Univ. Press (1959).

[9] J. V. Evans, S. Evans, and J. H. Thomson, The rapid fading of moon echoes at $100 \mathrm{Mc} / \mathrm{s}$, Paris Symp. on Radio Astron. (ed. R. N. Bracewell), Stanford Univ. Press (1959).

[10] W. A. S. Murray and J. K. Hargreaves, Lunar radio echoes and the Faraday effect in the ionosphere, Nature 173, 944 (1954).

[11] B. C. Blevis, personal communications (8 October 1957 and 3 June 1958).

[12] J. Chapman, personal communications (15 to 22 October 1959).

[13] J. S. Hey and V. A. Hughes, Radar observations of the moon at $10 \mathrm{~cm}$ wavelength, Paris Symp. on Radio Astron. (ed. R. N. Bracewell), Stanford Univ. Press (1959).

[14] B. S. Yaplee, N. G. Roman, K. J. Craig, and T. F. Scanlan, A lunar radar study at $10 \mathrm{~cm}$ wavelength, Paris Symp. on Radio Astron. (ed. R. N. Bracewell) Stanford Univ. Press (1959).

[15] A. B. Youmans, Personal communication (6 June 1958).

[16] J. Aarons, H. Peters, and H. Whitney, Personal communications (29 to 30 October 1959).

[17] V. A. Hughes, Personal communication (28 April 1958).

[18] S. J. Fricker, R. P. Ingalls, W. C. Mason, and M. L. Stone, UHF moon reflections, Presented at URSI-IRE meeting, Washington, D.C. (1958).

[19] R.L. Leadabrand, Personal communication (30 October 1959).

[20] H. D. Webb, Personal communication (30 October 1959).

[21] J. V. Evans, The scattering of radio waves by the moon, Proc. Phys. Soc. B 70, 1105 (1957).

[22] V. H. Weston, Exact near field and far field solution for the backscattering of a pulse from a perfectly conducting sphere, Univ. of Michigan Radiation Lab. Rept. No. 2778-4-T (1959).

[23] J. A. Stratton, Electromagnetic Theory (McGraw-Hill Book Co., Inc., New York, N.Y., 1941).

[24] W. E. Fensler, T.B.A. Senior, and K. M. Siegel, Exploring the depth of the surface layer of the moon from a radar space observatory, Aero/Space Eng. 18, 38 (1959).

[25] E. M. Purcell, Personal communication (15 October 1959).

\section{Appendix: A Radar Cross Section Theorem}

Consider a body on which is incident a field whose direction and polarization are fixed in space. The scattered field is received with two orthogonally polarized antennas which are located at a fixed point in space, but whose polarization is variable. To begin with, it will be assumed that these antennas are in the far field of the body.

At any point in space the scattered field can be written as

$$
\underline{\mathrm{E}}^{s}=L^{\prime} \hat{r}+M^{\prime} \hat{\theta}+N^{\prime} \hat{\phi}
$$


where $\hat{r}, \hat{\theta}, \hat{\phi}$ are unit vectors for a system of spherical polar coordinates whose origin is in the neighborhood of the body. $L^{\prime}, M^{\prime}, N^{\prime}$ are functions of the incident field (in direction and polarization), the body (in shape and aspect), and the position of the receiving point. If this point is in the far field of the body, then

$$
\begin{aligned}
& \lim _{r \rightarrow \infty} L^{\prime}=\mathrm{O}\left(1 / r^{2}\right) \\
& \lim _{r \rightarrow \infty} M^{\prime}=\frac{e^{i k r}}{r} M+\mathrm{O}\left(1 / r^{2}\right) \\
& \lim _{r \rightarrow \infty} N^{\prime}=\frac{e^{i k r}}{r} N+\mathrm{O}\left(1 / r^{2}\right)
\end{aligned}
$$

where $M$ and $N$ are independent of $r$, a time dependence $e^{-i \omega t}$ having been assumed. Hence

with

$$
\underline{\mathrm{E}}^{s} \sim \frac{e^{i k r}}{r} \underline{\mathrm{F}}
$$

$$
\underline{\mathrm{F}}=M \theta+N \hat{\phi}
$$

For a receiving antenna which is polarized in the direction $\hat{x}$, where $\hat{x}$ makes an angle $\beta$ with some fixed direction, the cross section which is measured is

$$
\sigma(\alpha, \beta)=4 \pi|\underline{\mathrm{F}} \cdot \hat{x}|^{2}
$$

and this can be written in the form

$$
\sigma(\alpha, \beta)=4 \pi|M \cos \Omega+N \sin \Omega|^{2}
$$

where $\Omega$ is the angle between the projection of $\hat{x}$ in the $(\theta, \phi)$ plane and the vector $\hat{\phi}$. Similarly, for the receiving antenna which is orthogonally polarized, the cross section is

$$
\sigma\left(\alpha, \beta+\frac{\pi}{2}\right)=4 \pi|M \sin \Omega-N \cos \Omega|^{2}
$$

and hence

$$
\sigma(\alpha, \beta)+\sigma\left(\alpha, \beta+\frac{\pi}{2}\right)=4 \pi\left(|M|^{2}+|N|^{2}\right) .
$$

For a fixed transmitted field and for a fixed location of the receiving antennas, the right-hand side of this equation is at most a function of the aspect of the body (which can be changed by rotating the body keeping the antennas fixed). In particular, it is independent of $\Omega$ (and therefore $\beta$ ), and in consequence it holds for all receiver polarizations.

If the receiving antennas are not in the far field, only a trivial modification of this proof is required. The field component $L^{\prime}$ must now be retained, and the equation corresponding to (A.1) is then

$\sigma(\alpha, \beta)+\sigma\left(\alpha, \beta+\frac{\pi}{2}\right)=\lim _{r \rightarrow \infty} 4 \pi r^{2}\left(\left|L^{\prime}\right|^{2}+\left|M^{\prime}\right|^{2}+\left|N^{\prime}\right|^{2}\right)$.

To the first order in $r$ this is identical to eq (A.1)

Boulder, Colo.

(Paper 64D3-51) 